\title{
On the adjacency matrix of a block graph
}

\author{
R. B. Bapat \\ Stat-Math Unit \\ Indian Statistical Institute, Delhi \\ 7-SJSS Marg, New Delhi 110 016, India. \\ email: rbb@isid.ac.in \\ Souvik Roy \\ Economics and Planning Unit \\ Indian Statistical Institute, Delhi \\ 7-SJSS Marg, New Delhi 110 016, India. \\ email: souvik_roy2004@yahoo.com
}

February 1, 2013

\begin{abstract}
A block graph is a graph in which every block is a complete graph. Let $G$ be a block graph and let $A$ be the adjacency matrix of $G$. We first obtain a formula for the determinant of $A$ over reals. It is shown that $A$ is nonsingular over $\mathbb{F}_{2}$ if and only if the removal of any vertex from $G$ produces a graph with exactly one odd component. A formula for the inverse of $A$ over $\mathbb{F}_{2}$ is obtained, whenever it exists. We obtain some results for the adjacency matrices over $\mathbb{F}_{2}$, of claw-free block graphs, which are the same as the line graphs of trees, and for the adjacency matrices of flowers, which are block graphs with only one cut-vertex.
\end{abstract}

Key words. block graph, adjacency matrix, determinant, tree, line graph

AMS Subject Classifications. 15A15, 05C05. 


\section{Introduction}

We consider undirected graphs with no loops or parallel edges. We refer to [11] for graph theoretic preliminaries. Recall that a block in a graph is a maximal connected subgraph that has no cut-vertex ([11],p.15). The complete graph with $n$ vertices is denoted by $K_{n}$. A block graph is a graph in which each block is a complete graph. A block graph whose blocks are $K_{2}, K_{2}, K_{3}, K_{4}$ and $K_{5}$ is shown below.

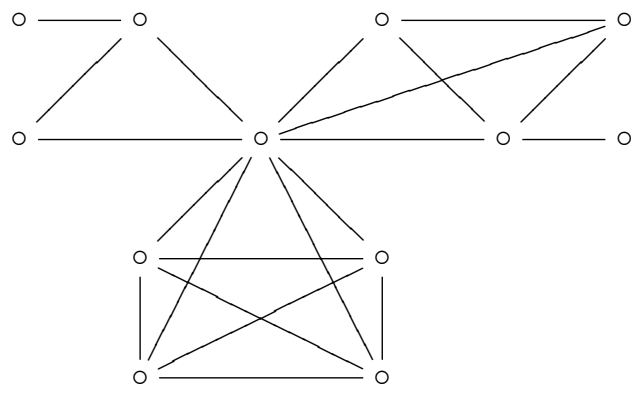

We consider the adjacency matrix of a block graph over the field of reals as well as over the two-element field $\{0,1\}$, which we denote as $\mathbb{F}_{2}$. For basic definitions concerning matrices associated with graphs, see [1].

A graph is said to be nonsingular over reals if its adjacency matrix is nonsingular over reals. Similarly, a graph is said to be nonsingular over $\mathbb{F}_{2}$ if its adjacency matrix is nonsingular over $\mathbb{F}_{2}$. It is well-known that a tree is nonsingular over reals if and only if it has a perfect matching. Moreover, when a tree is nonsingular over reals, there is a formula for its inverse in terms of alternating paths, see [3],[4], and the references contained therein. These two results motivated this work. Since a tree is a block graph, it is natural to investigate the adjacency matrix of a general block graph.

We now describe the contents of this paper. Let $G$ be a block graph and let $A$ be the adjacency matrix of $G$. In Section 2 we obtain a formula for the determinant of $A$ over reals. As a corollary we obtain a sufficient condition for the determinant to be an even integer. In Sections 3,4 and 5 we work over $\mathbb{F}_{2}$. In Section 3 we consider the adjacency matrix of a block graph over $\mathbb{F}_{2}$, It is shown that $A$ is nonsingular over $\mathbb{F}_{2}$ if and only if the removal of any vertex from $G$ produces a graph with exactly one odd component. A formula for the inverse of $A$ over $\mathbb{F}_{2}$ is obtained, whenever it exists. In the final two sections we illustrate applications of our results to two special classes of block graphs. In Section 4 we obtain some results for the line graph of a tree, which is a block graph in which every cut-vertex is incident to at most two blocks. A flower, which is a block graph with only one cut-vertex is considered in Section 5 . 


\section{Adjacency matrix of a block graph over reals}

A pendant vertex is a vertex of degree 1. A block is called a pendant block if it has only one cut-vertex or if it is the only block in that component. A graph is called even (odd) if it has an even (odd) number of vertices. An isolated vertex in a graph is considered to be a block of the graph. The vertex set of the graph $G$ is denoted by $V(G)$. The transpose of the matrix $A$ is denoted $A^{\prime}$. Let $G$ be a block graph and let $B_{1}, \ldots, B_{k}$ be the blocks of $G$. If $S \subset\{1, \ldots, k\}$, then $G_{S}$ will denote the subgraph of $G$ induced by the blocks $B_{i}, i \in S$. We first prove a preliminary result.

Lemma 1 Let $G$ be a block graph with $n$ vertices. Let $B_{1}, \ldots, B_{k}$ be the blocks of $G$ where $B_{i}$ is the complete graph with $b_{i}$ vertices, $i=1, \ldots, k$. Let $\left(\alpha_{1}, \ldots, \alpha_{k}\right)$ be a $k$-tuple of nonnegative integers satisfying the following conditions:

(i) $\sum_{i=1}^{k} \alpha_{i}=n$

(ii) for any nonempty $S \subset\{1, \ldots, k\}$,

$$
\sum_{i \in S} \alpha_{i} \leq\left|V\left(G_{S}\right)\right|
$$

If $B_{i}$ is a pendant block, then $\alpha_{i}$ equals either $b_{i}$ or $b_{i}-1$.

Proof: Clearly by (1), with $S=\{i\}$, we must have $0 \leq \alpha_{i} \leq b_{i}$. Setting $S=\{1, \ldots, k\} \backslash\{i\}$ in (1) we see from (i) and (ii) that $\alpha_{1}+\cdots+\alpha_{n}-\alpha_{i}=n-\alpha_{i} \leq n-b_{i}+1$, and hence $\alpha_{i} \geq b_{i}-1$. That completes the proof.

In the next result, which is the main result of this section, we obtain a formula for the determinant of the adjacency matrix of a block graph over reals.

Theorem 2 Let $G$ be a block graph with $n$ vertices. Let $B_{1}, \ldots, B_{k}$ be the blocks of $G$. Let $A$ be the adjacency matrix of $G$. Then

$$
\operatorname{det} A=(-1)^{n-k} \sum\left(\alpha_{1}-1\right) \cdots\left(\alpha_{k}-1\right)
$$

where the summation is over all $k$-tuples $\left(\alpha_{1}, \ldots, \alpha_{k}\right)$ of nonnegative integers satisfying the following conditions:

(i) $\sum_{i=1}^{k} \alpha_{i}=n$

(ii) for any nonempty $S \subset\{1, \ldots, k\}$,

$$
\sum_{i \in S} \alpha_{i} \leq\left|V\left(G_{S}\right)\right|
$$


Proof: We prove the result by induction on $k$. Note that the result is true for a block graph with one block, since the adjacency matrix of the complete graph $K_{p}$ has determinant $(-1)^{p-1}(p-1)$. Assume the result to be true for a block graph with at most $k-1$ blocks and proceed. We consider two cases.

Case (i): The graph $G$ has a pendant block with exactly 2 vertices.

Let $B_{1}=K_{2}$ be a pendant block in $G$. Let the vertices of $B_{1}$ be 1 and 2 , where 1 is pendant. We assume that the vertex 2 is in blocks $B_{1}, \ldots, B_{p}$ and we further assume that among these blocks, the first $q$ blocks $B_{1}, \ldots, B_{q}$ are equal to $K_{2}$. It is possible that $q=1$. Let $G_{1}=G \backslash 1$. The matrix $A$ has the form

$$
A=\left(\begin{array}{ccccc}
0 & 1 & 0 & \cdots & 0 \\
1 & 0 & & \cdots & \\
0 & & & & \\
\vdots & \vdots & & A_{1} & \\
0 & & & &
\end{array}\right)
$$

where $A_{1}$ is the adjacency matrix of $G_{2}=G_{1} \backslash 2$. It follows from (4) that $\operatorname{det} A=-\operatorname{det} A_{1}$.

The graph $G_{2}$ has $k-q$ blocks given by $C_{i}, i=q+1 \ldots, k$, defined as follows: $C_{i}=$ $B_{i} \backslash 2, i=q+1, \ldots, p$ and $C_{i}=B_{i}, i=p+1, \ldots, k$.

First consider the case when $q>1$ and that there is at least one pendant block among $B_{2}, \ldots, B_{q}$. let us assume, without loss of generality, that $B_{2}$ is a pendant block. We also assume that $V\left(B_{2}\right)=\{2,3\}$. Then the first and the third columns of $A$ are identical and $\operatorname{det} A=0$. Also if $\left(\alpha_{1}, \ldots, \alpha_{k}\right)$ is a $k$-tuple of nonnegative integers satisfying the conditions in the Theorem, then at least one of $\alpha_{1}$ or $\alpha_{2}$ must equal 1 and hence the summation in (2) is zero. Therefore the result is proved in this case. We therefore assume that none of the blocks among $B_{2}, \ldots, B_{q}$ is pendant.

Consider

$$
(-1)^{n-k} \sum\left(\alpha_{1}-1\right) \cdots\left(\alpha_{k}-1\right)
$$

where the summation is over all $k$-tuples $\left(\alpha_{1}, \ldots, \alpha_{k}\right)$ of nonnegative integers satisfying the following conditions:

(i) $\sum_{i=1}^{k} \alpha_{i}=n$

(ii) for any nonempty $S \subset\{1, \ldots, k\}$,

$$
\sum_{i \in S} \alpha_{i} \leq\left|V\left(G_{S}\right)\right|
$$

By Lemma $1, \alpha_{1}$ equals either 1 or 2 . If $\alpha_{1}=1$, then the corresponding term in (5) is zero, so we assume $\alpha_{1}=2$. Then it follows from (ii) that none of $\alpha_{2}, \ldots, \alpha_{q}$ can be 2 . For 
example, if $\alpha_{2}=2$, then $\alpha_{1}+\alpha_{2}=4$, whereas the graph induced by blocks $B_{1}$ and $B_{2}$ has 3 vertices, thus violating (6). Thus each of $\alpha_{2}, \ldots, \alpha_{q}$ is either 0 or 1 , and again we may assume that $\alpha_{2}=\cdots=\alpha_{q}=0$. In view of these observations (5) equals

$$
(-1)^{n-k}(-1)^{q-1} \sum\left(\alpha_{q+1}-1\right) \cdots\left(\alpha_{k}-1\right)=-(-1)^{n-2-(k-q)} \sum\left(\alpha_{q+1}-1\right) \cdots\left(\alpha_{k}-1\right) .
$$

The summation in $(7)$ is over all $(k-q)$-tuples $\left(\alpha_{q+1}, \ldots, \alpha_{k}\right)$ of nonnegative integers satisfying the following conditions:

(i) $\sum_{i=q+1}^{k} \alpha_{i}=n-2$

(ii) for any nonempty $S \subset\{q+1, \ldots, k\}$,

$$
\sum_{i \in S} \alpha_{i} \leq\left|V\left(G_{S}\right)\right|
$$

By the induction assumption, the right side of (7) equals $-\operatorname{det} A_{1}$ and $\operatorname{since} \operatorname{det} A=$ - $\operatorname{det} A_{1}$, it follows that $\operatorname{det} A$ equals (5). Therefore the proof is complete in this case.

Case (ii): The graph $G$ does not have a pendant block with exactly 2 vertices.

Let $B_{1}$ be a pendant block of $G$ and let $V\left(B_{1}\right)=\left\{1, \ldots, b_{1}\right\}, b_{1} \geq 3$. Let $b_{1}$ be the cut vertex in $B_{1}$. Let $H=G \backslash\left\{B_{1} \backslash b_{1}\right\}$ be the block graph with $n-b_{1}+1$ vertices having blocks $B_{2}, \ldots, B_{k}$ and let $A_{1}$ be the adjacency matrix of $H$.

After a suitable relabeling of the vertices in $G$ we may write

$$
A=\left(\begin{array}{cc}
D & C \\
C^{\prime} & A_{1}
\end{array}\right)
$$

where $D$ is the adjacency matrix of the subgraph induced by the vertex set $V\left(B_{1} \backslash b_{1}\right), C$ is a $\left(b_{1}-1\right) \times\left(n-b_{1}+1\right)$ matrix with $c_{i j}=1$ if $j=b_{1}+1$, and $c_{i j}=0$ otherwise. Thus $D=J-I$ where $J$ is the matrix of all ones and $C$ has the form

$$
\left(\begin{array}{cccc}
1 & 0 & \cdots & 0 \\
\vdots & \vdots & & \vdots \\
1 & 0 & \cdots & 0
\end{array}\right) .
$$

Note that the first row of $A_{1}$ corresponds to the vertex $b_{1}$.

By the Schur complement formula ([1],p.4) we have

$$
\operatorname{det} A=\operatorname{det} D \cdot \operatorname{det}\left(A_{1}-C^{\prime} D^{-1} C\right) .
$$

We note the following simple facts:

(i) $\operatorname{det} D=(-1)^{b_{1}-2}\left(b_{1}-2\right)$ 
(ii) $D^{-1}=\frac{1}{b_{1}-2} J-I$

(iii) The matrix $C^{\prime} D^{-1} C$ has all entries zero except the entry in the first row and the first column, which is $(-1)^{b_{1}} \frac{b_{1}-1}{\operatorname{det} D}$.

Let $M$ be the adjacency matrix of $H \backslash b_{1}$. Thus $M$ is the submatrix of $A_{1}$ formed by its last $n-b_{1}$ rows and columns. It follows from (i)-(iii) that

$$
\operatorname{det}\left(A_{1}-C^{\prime} D^{-1} C\right)=\operatorname{det} A_{1}-\frac{b_{1}-1}{\operatorname{det} D} \operatorname{det} M .
$$

From (10),(11) we have

$$
\begin{aligned}
\operatorname{det} A & =(\operatorname{det} D)\left(\operatorname{det} A_{1}-(-1)^{b_{1}} \frac{b_{1}-1}{\operatorname{det} D} \operatorname{det} M\right) \\
& =(\operatorname{det} D)\left(\operatorname{det} A_{1}\right)-(-1)^{b_{1}}\left(b_{1}-1\right) \operatorname{det} M \\
& =(-1)^{b_{1}-2}\left(b_{1}-2\right) \operatorname{det} A_{1}-(-1)^{b_{1}}\left(b_{1}-1\right) \operatorname{det} M .
\end{aligned}
$$

We assume that $b_{1}$ is in blocks $B_{1}$ and $B_{2}, \ldots, B_{p}$. If there are any blocks equal to $K_{2}$ containing $b_{1}$, then we enumerate them as $B_{2}, \ldots, B_{q}$. The remaining blocks, which do not contain $b_{1}$ are $B_{p+1}, \ldots, B_{k}$. If there are no blocks equal to $K_{2}$ containing $b_{1}$, then we set $q=1$.

The graph $H \backslash b_{1}$ has $k-q$ blocks given by $C_{i}, i=q+1 \ldots, k$, defined as follows: $C_{i}=B_{i} \backslash b_{1}, i=q+1, \ldots, p$ and $C_{i}=B_{i}, i=p+1, \ldots, k$.

Consider

$$
\sum(-1)^{n-k}\left(\alpha_{1}-1\right) \cdots\left(\alpha_{k}-1\right)
$$

where the summation is over all $k$-tuples $\left(\alpha_{1}, \ldots, \alpha_{k}\right)$ of nonnegative integers satisfying the following conditions:

(i) $\sum_{i=1}^{k} \alpha_{i}=n$

(ii) for any nonempty $S \subset\{1, \ldots, k\}$,

$$
\sum_{i \in S} \alpha_{i} \leq\left|V\left(G_{S}\right)\right|
$$

By Lemma 1, $\alpha_{1}$ equals either $b_{1}$ or $b_{1}-1$. If $\alpha_{1}=b_{1}$, then each of $\alpha_{2}, \ldots, \alpha_{q}$ must be either 0 or 1 . For example, if $\alpha_{2}=2$, then $\alpha_{1}+\alpha_{2}=b_{1}+2$, whereas the graph induced by blocks $B_{1}$ and $B_{2}$ has $b_{1}+1$ vertices, thus violating (6). If $\alpha_{i}=1$ for some $i \in\{2, \ldots, q\}$, then the corresponding term in (13) is zero and hence we assume $\alpha_{2}=\cdots=\alpha_{q}=0$. Then it can be seen by the induction hypothesis, that the sum of the terms in (13) corresponding to $\alpha_{1}=b_{1}$ equals $-(-1)^{b_{1}}\left(b_{1}-1\right) \operatorname{det} M$. 
Similarly, by the induction hypothesis, the sum of the terms in (13) corresponding to $\alpha_{1}=b_{1}-1$ equals $(-1)^{b_{1}-2}\left(b_{1}-2\right) \operatorname{det} A_{1}$. It follows that the sum in (13) equals

$$
(-1)^{b_{1}-2}\left(b_{1}-2\right) \operatorname{det} A_{1}-(-1)^{b_{1}}\left(b_{1}-1\right) \operatorname{det} M
$$

which is $\operatorname{det} A$ by (12). That completes the proof.

As noted in the introduction, it is well-known that a tree is nonsingular over reals if and only if it has a perfect matching. We now derive this result from Theorem 2.

Corollary 3 Let $T$ be a tree with $n$ vertices and let $A$ be the adjacency matrix of $T$. Then $A$ is nonsingular over reals if and only if $T$ has a perfect matching.

Proof: First suppose that $A$ is nonsingular. Then at least one term in the summation in (2) must be nonzero. If $\left(\alpha_{1}, \ldots, \alpha_{n-1}\right)$ is an $(n-1)$-tuple of nonnegative integers satisfying $(i)$ and (ii) of Theorem 2 and if the corresponding term in (2) is nonzero, then each $\alpha_{i}$ is either 0 or 2 . Moreover if two edges have a common vertex, then the corresponding $\alpha$ 's cannot both be nonzero, in view of (ii). Thus there must be a perfect matching in $T$ and $\alpha_{i}=2$ if and only if the corresponding edge is in the matching.

Conversely, suppose $T$ has a perfect matching. As noted in the first part of the proof, a nonzero term in the summation in (2) corresponds to a perfect matching. We invoke the elementary fact, easily proved by induction, that if a tree has a perfect matching then it must be unique. Thus there must be precisely one nonzero term in the summation in (2) which renders $\operatorname{det} A$ nonzero. Thus $A$ is nonsingular over reals and the proof is complete.

Characterizing block graphs, other than trees, which are nonsingular over reals seems to be an interesting open problem. One example of a singular block graph is a tree with no perfect matching. There are other examples. The following block graph is singular since the adjacency matrix has two identical columns corresponding to the pendant vertices.

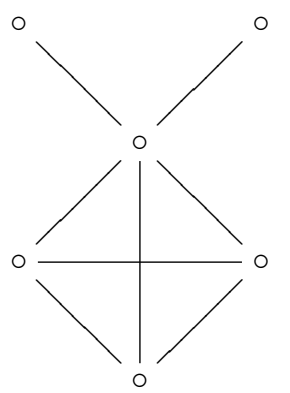

In the following corollary we identify a class of graphs whose determinant is even. The result will be used in the next section when we consider the adjacency matrix over $\mathbb{F}_{2}$. 
Corollary 4 Let $G$ be a connected block graph with $n$ vertices. Let $v$ be a vertex of $G$ and let $H_{1}, \ldots, H_{\ell}$ be the components of $G \backslash v$. Let us suppose that among $\left|V\left(H_{i}\right)\right|, i=1, \ldots, \ell$, at least two integers are odd. Then $\operatorname{det} A$ is even.

Proof: Let us list the blocks of $G$ as follows. Let $B_{1}^{i}, \ldots, B_{m_{i}}^{i}$ be the blocks of $H_{i} \cup v, i=$ $1, \ldots, \ell$. Let $\alpha_{1}^{i}, \ldots, \alpha_{m_{i}}^{i}, i=1, \ldots, \ell$ be integers satisfying the conditions of Theorem 2 . Let $B_{j}^{i}$ have $p_{j}^{i}$ vertices, $i=1, \ldots, \ell ; j=1, \ldots m_{i}$. Then

$$
\sum_{j=1}^{m_{i}} \alpha_{j}^{i} \leq\left|V\left(H_{i}\right)\right|+1, i=1, \ldots, \ell .
$$

It follows that

$$
\sum_{i=1}^{\ell} \sum_{j=1}^{m_{i}} \alpha_{j}^{i} \leq \sum_{i=1}^{\ell}\left(\left|V\left(H_{i}\right)\right|+1\right), i=1, \ldots, \ell
$$

Also

$$
\sum_{i=1}^{\ell} \sum_{j=1}^{m_{i}} \alpha_{j}^{i}=n=\sum_{i=1}^{\ell}\left(\left|V\left(H_{i}\right)\right|+1\right)-(\ell-1) .
$$

If among the $\ell$ inequalities in (15) some two are equalities, then it will violate the condition in Theorem 2 (by considering $H_{i} \cup H_{j} \cup v$ for those $i, j$.) If all the $\ell$ inequalities in (15) are strict, then we get a contradiction as follows. If

$$
\sum_{j=1}^{m_{i}} \alpha_{j}^{i} \leq\left|V\left(H_{i}\right)\right|, i=1, \ldots, \ell,
$$

then

$$
\sum_{i=1}^{\ell} \sum_{j=1}^{m_{i}} \alpha_{j}^{i} \leq \sum_{i=1}^{\ell}\left(\left|V\left(H_{i}\right)\right|+1\right)-\ell
$$

From (18) and (19) we get $\ell \leq \ell-1$, a contradiction. For a similar reason, in any inequality, the deficiency is 1 . We conclude that among the $\ell$ inequalities in (15), $\ell-1$ must be strict (with deficiency 1) and one must be equality. Without loss of generality, we assume

$$
\sum_{j=1}^{m_{1}} \alpha_{j}^{1}=\left|V\left(H_{1}\right)\right|+1,
$$

and

$$
\sum_{j=1}^{m_{i}} \alpha_{j}^{i}=\left|V\left(H_{i}\right)\right|, i=2, \ldots, \ell .
$$

According to our assumption, among $\left|V\left(H_{i}\right)\right|, i=1, \ldots, \ell$, at least two integers are odd and hence, in view of (20),(21), there exists $s \in\{2, \ldots, \ell\}$ such that

$$
\sum_{j=1}^{m_{s}} \alpha_{j}^{s}=\left|V\left(H_{s}\right)\right|=1 \bmod 2 .
$$

Therefore at least one integer among $\alpha_{1}^{s}, \ldots, \alpha_{m_{s}}^{s}$ must be odd and the corresponding term in the summation in Theorem 2 is even. Thus each term in the summation in Theorem 2 is even and the result follows. 


\section{Adjacency matrix of a block graph over $\mathbb{F}_{2}$}

We first prove a preliminary result.

Lemma 5 Let $G$ be a graph with $n$ vertices and let $A$ be the adjacency matrix of $A$ over reals. If $n$ is odd, then $\operatorname{det} A$ is an even integer. In particular, $A$ is singular over $\mathbb{F}_{2}$.

Proof: We have

$$
\operatorname{det} A=\sum_{\sigma}(-1)^{\epsilon(\sigma)} a_{1 \sigma(1)} \cdots a_{n \sigma(n)},
$$

where $\epsilon(\sigma)$ is $1(-1)$ if $\sigma$ is even(odd), and where the summation is over all permutations $\sigma$ of $1, \ldots, n$. Consider a permutation $\sigma$ for which $a_{1 \sigma(1)} \cdots a_{n \sigma(n)}$ is nonzero. Since the diagonal elements of $A$ are zero and since $n$ is odd, in the cycle decomposition of $\sigma$, there must be an odd cycle of length greater than one. Thus if $\tau=\sigma^{-1}$, then $\tau \neq \sigma$ and $a_{1 \tau(1)} \cdots a_{n \tau(n)}$ is nonzero as well. Therefore we may group the nonzero terms in the summation in (23) in pairs and hence $\operatorname{det} A$ is even.

Theorem 6 Let $G$ be a graph with $n$ vertices and let $A$ be the adjacency matrix of $G$. Then $A$ is nonsingular over $\mathbb{F}_{2}$ if and only if $n$ is even and for all $u \in V(G), G \backslash u$ has exactly one odd component.

Proof: First suppose that $A$ is nonsingular over $\mathbb{F}_{2}$. By Lemma $5, n$ is an even integer. Also if there exists $u \in V(G)$ such that $G \backslash u$ has at least two odd components, then by Corollary $4, \operatorname{det} A$, over reals, is an even integer and hence $A$ must be singular over $\mathbb{F}_{2}$, which is a contradiction. This proves the "only if" part of the Theorem.

We turn to the "if" part. The proof will be by induction on the number of blocks. The result is easily verified when $G$ has a single block. Let $G$ be a graph with $k$ blocks. Assume the result to be true for a graph with at most $k-1$ blocks and proceed. Let $G$ have $n$ vertices where $n$ is even and suppose that for all $u \in V(G), G \backslash u$ has exactly one odd component. Let $B$ be a pendant block in $G$ with cut-vertex $t$. We distinguish two cases.

Case 1. $|V(B)|$ is odd. Note that the graph $G \backslash(B \backslash t)$ also satisfies the conditions of the theorem. After a suitable relabeling of the vertices in $G$ we may write

$$
A=\left(\begin{array}{cc}
D & C \\
C^{\prime} & A_{1}
\end{array}\right)
$$

where $D$ is the adjacency matrix of the subgraph induced by the vertex set $V(B \backslash t)$ and $C$ is a $(t-1) \times(n-t+1)$ matrix with $c_{i j}=1$ if $j=t+1$, and $c_{i j}=0$ otherwise. Thus $D=J-I$ where $J$ is the matrix of all ones and $C$ has the form

$$
\left(\begin{array}{cccc}
1 & 0 & \cdots & 0 \\
\vdots & \vdots & & \vdots \\
1 & 0 & \cdots & 0
\end{array}\right) .
$$


Note that the first row of $A_{1}$ corresponds to the vertex $t$. Perform the following row and column operations on $A$ : Add the first $t-1$ columns (rows) to the column (row) $t$. Then the resulting matrix $\tilde{A}$ has the form

$$
\tilde{A}=\left(\begin{array}{cc}
D & 0 \\
0 & A_{1}
\end{array}\right) .
$$

Note that $D=J-I$ being of even order, is nonsingular over $\mathbb{F}_{2}$. Since $G \backslash(B \backslash t)$ also satisfies the conditions of the theorem, by the induction hypothesis, its adjacency matrix $A_{1}$ is nonsingular over $\mathbb{F}_{2}$. It follows from $(25)$ that $\tilde{A}$ is nonsingular over $\mathbb{F}_{2}$. Since $\tilde{A}$ and $A$ have the same determinant, it follows that $A$ is nonsingular over $\mathbb{F}_{2}$.

Case 2. $|V(B)|$ is even. Note that the graph $G \backslash B$ also satisfies the conditions of the theorem. After a suitable relabeling of the vertices in $G$ we may write

$$
A=\left(\begin{array}{cc}
D & C \\
C^{\prime} & A_{1}
\end{array}\right)
$$

where $D=J-I$ is the adjacency matrix of $B, A_{1}$ is the adjacency matrix of $G \backslash B$, and $C$ is a $t \times(n-t)$ matrix with $c_{i j}=0$ if $i=1,2, \ldots, t-1$. Perform the following row and column operations on $A$ : Add the first $t-2$ columns (rows) to the column (row) $t-1$. Evaluate the determinant of the resulting matrix by Laplace expansion with respect to column $t$, followed by Laplace expansion with respect to row $t$. Then it is seen that $\operatorname{det} A=\operatorname{det} A_{1}$. Since $G \backslash B$ satisfies the conditions of the theorem, by the induction hypothesis, its adjacency matrix $A_{1}$ is nonsingular over $\mathbb{F}_{2}$. It follows that $A$ is nonsingular over $\mathbb{F}_{2}$ and the proof is complete.

We record the following consequence of Theorem 6 .

Corollary 7 Let $G$ be a graph, let $B$ be a pendant block of $G$, and let $t$ be the cut-vertex in $B$. If $|V(B)|$ is odd, then $G$ is nonsingular over $\mathbb{F}_{2}$ if and only if $G \backslash(B \backslash t)$ is nonsingular. If $|V(B)|$ is even, then $G$ is nonsingular over $\mathbb{F}_{2}$ if and only if $G \backslash B$ is nonsingular.

Proof: We first consider the case when $|V(B)|$ is odd. Let $G$ be nonsingular. By Theorem 6 , for any $u \in V(G), G \backslash u$ has exactly one odd component. We claim that for any $u \in$ $V(G \backslash(B \backslash t))$, the graph $(G \backslash(B \backslash t)) \backslash u$ has exactly one odd component. Consider two subcases.

Subcase (i), $u=t$ : If $(G \backslash(B \backslash t) \backslash t$ has more than one odd components, they continue to be odd components of $G \backslash t$, which is a contradiction.

Subcase (ii), $u \neq t$ : If $(G \backslash(B \backslash t)) \backslash u$ has more than one odd components, denote two such components by $\mathcal{O}_{1}$ and $\mathcal{O}_{2}$. If $t \notin \mathcal{O}_{1} \cup \mathcal{O}_{2}$, then $\mathcal{O}_{1}$ and $\mathcal{O}_{2}$ are odd components of $G \backslash t$, which is a contradiction. If $t \in \mathcal{O}_{1}$, then again $\mathcal{O}_{1} \cup B$ and $\mathcal{O}_{2}$ are odd components in $G \backslash u$, which is a contradiction. A similar conclusion holds if $t \in \mathcal{O}_{2}$. 
Thus the claim is proved and by Theorem $6, G \backslash(B \backslash t)$ is nonsingular. This completes the proof of the "only if" part. Conversely, suppose $G \backslash(B \backslash t)$ is nonsingular. Then for any $u \in V(G \backslash(B \backslash t))$, the graph $(G \backslash(B \backslash t)) \backslash u$ has exactly one odd component. Using a similar argument as before, we can show that for any $u \in V(G), G \backslash u$ has exactly one odd component, and by Theorem $6, G$ is nonsingular.

We omit the proof in the case when $|V(B)|$ is even, since it is similar.

As noted in Corollary 3, a tree is nonsingular over reals if and only if it has a perfect matching. Since the determinant over reals of the adjacency matrix of a tree is either 0 or \pm 1 , it follows that a tree is nonsingular over $\mathbb{F}_{2}$ if and only if it has a perfect matching. It may be instructive to note the connection between existence of a perfect matching in a tree and the hypothesis in Theorem 6 . Let $T$ be a tree with a perfect matching. Let $v \in V(T)$ be of degree $k$, and let it be adjacent to $v_{1}, \ldots, v_{k}$. Let $T_{1}, \ldots, T_{k}$ be the components of $T \backslash v$, containing $v_{1}, \ldots, v_{k}$ respectively. Since $T$ has a perfect matching, there exists a unique $i \in\{1, \ldots, k\}$ such that the edge $v v_{i}$ is in the matching. Then for any $j \neq i, T_{j}$ has a perfect matching and must be an even component. Thus $T_{i}$ is the unique odd component in $T \backslash v$ and the hypothesis in Theorem 6 holds.

If $A$ is an $n \times n$ matrix, we denote by $A(i \mid j)$, the submatrix obtained by deleting row $i$ and column $j$. Similarly, $A(i, j \mid i, j)$ will denote the submatrix obtained by deleting rows $i, j$ and columns $i, j$.

Theorem 8 Let $G$ be a graph with $n$ vertices and let $A$ be the adjacency matrix of $G$. Let $A$ be nonsingular over $\mathbb{F}_{2}$ and let $B=A^{-1}$. Then $b_{i i}=0, i=1, \ldots, n$. Moreover, if $i \neq j$ are vertices of $G$, then the following conditions are equivalent:

(i) $b_{i j}=1$

(ii) $\operatorname{det} A(i \mid j)=1$

(iii) $\operatorname{det} A(i, j \mid i, j)=1$

(iv) $G \backslash\{i, j\}$ is nonsingular over $\mathbb{F}_{2}$

(v) For any vertex $k$, not equal to $i, j, G \backslash\{i, j, k\}$ has exactly one odd component.

Proof: Since $A$ is nonsingular over $\mathbb{F}_{2}$, by Lemma $5, n$ is even. For any $i, G \backslash\{i\}$ has an odd number of vertices and hence it is singular over $\mathbb{F}_{2}$ by Lemma 5 . Therefore $\operatorname{det} A(i \mid i)=0$ and hence $b_{i i}=0, i=1, \ldots, n$.

The equivalence of $(i),(i i)$ is obvious, and so is the equivalence of (iii) and (iv). By Theorem $6,(i v)$ and $(v)$ are equivalent. It remains to show the equivalence of $(i i)$ and $(i i i)$. We recall the well-known identity (which is a special case of Sylvester's identity, see [9],p.22): If $A$ is an $n \times n$ matrix, then for any $i \neq j$,

$$
\operatorname{det} A(i \mid i) \operatorname{det} A(j \mid j)-\operatorname{det} A(i \mid j) \operatorname{det} A(j \mid i)=(\operatorname{det} A) \operatorname{det} A(i, j \mid i, j) .
$$


In particular, in the present situation, $A$ is a matrix over $\mathbb{F}_{2}$, $\operatorname{det} A(i \mid i)=0$ for all $i$, and $\operatorname{det} A=1$. Hence it follows from (27) that $\operatorname{det} A(i \mid j)=\operatorname{det} A(i, j \mid i, j)$ and therefore $\operatorname{det} A(i \mid j)=1$ if and only if $\operatorname{det} A(i, j \mid i, j)=1$. Thus (ii) and (iii) are equivalent. That completes the proof.

\section{Line graph of a tree}

Let $T$ be a tree with $V(T)=\{1, \ldots, n\}$ and $E(T)=\left\{e_{1}, \ldots, e_{n-1}.\right\}$ Let $L(T)$ be the line graph of $T$. Thus $L(T)$ has vertex set $E(T)$ and two vertices are adjacent if the corresponding edges of $T$ have a vertex in common. It can be seen that $L(T)$ is a block graph with the additional property that any cut-vertex is in at most two blocks. Conversely, a connected block graph in which any cut-vertex is in at most two blocks is the line graph of a tree. Let $K_{1,3}$ be the complete bipartite graph with the two parts having 1 and 3 vertices, respectively. Recall that a graph is said to be claw-free if it does not contain $K_{1,3}$ as an induced subgraph. Then it can be seen that a block graph is the line graph of a tree if and only if it is claw-free. The next result is a simple consequence of Theorems 6 and 8 .

Theorem 9 Let $G$ be a claw-free block graph with $n$ vertices and let $A$ be the adjacency matrix of $G$. Then $A$ is nonsingular over $\mathbb{F}_{2}$ if and only if $n$ is even. Moreover let $B=A^{-1}$ in case $A$ is nonsingular. Then $b_{i i}=0, i=1, \ldots, n$, and for $1 \leq i \neq j \leq n, b_{i j}=1$ if and only if $L(T) \backslash\{i, j\}$ has no odd component.

Proof: If $n$ is odd, then by Lemma $5, A$ is singular. So suppose $n$ is even. Since $G$ is a block graph in which any cut-vertex is in at most two blocks, $G \backslash\{i\}$ has at most two components for any vertex $i$ of $G$. Since $G$ has an even number of vertices, $G \backslash\{i\}$ must have exactly one odd component, and by Theorem $6, A$ is nonsingular over $\mathbb{F}_{2}$.

If $B=A^{-1}$, then by Theorem $8, b_{i i}=0, i=1, \ldots, n$. Moreover, $b_{i j}=1$ if and only if $G \backslash\{i, j\}$ is a nonsingular graph over $\mathbb{F}_{2}$. Since each component of $G \backslash\{i, j\}$ is the line graph of a tree, by the first part, $G \backslash\{i, j\}$ is nonsingular over $\mathbb{F}_{2}$ if and only if it has no odd component. That completes the proof.

Theorem 10 Let $G$ be a claw-free block graph with $n$ vertices and let Let $A$ be the adjacency matrix of $G$. If $n$ is even, then $A$ is nonsingular over $\mathbb{F}_{2}$. If $n$ is odd, then $A$ has nullity 1 over $\mathbb{F}_{2}$.

Proof: If $n$ is even, then since $G$ is claw-free, it readily follows from Theorem 6 that $A$ is nonsingular.

Now assume that $n$ is odd. Let $x$ be the incidence vector of the vertices $i$ such that $G \backslash\{i\}$ has no odd component. We first show that $x$ satisfies $A x=0$. Let $i$ be a vertex of $G$ with degree $r$ and let $i$ be adjacent to vertices $i_{1}, \ldots, i_{r}$. We further assume that $G \backslash\left\{i_{j}\right\}$ 
has no odd component if and only if $j=1, \ldots, s$. By the hypothesis, $G \backslash\left\{i_{j}\right\}$ has at most two components and we set $\mathcal{C}_{j}$ to be the component, which may be vacuous, that does not contain $i, j=1, \ldots, r$. Note that $\mathcal{C}_{j}$ and $\mathcal{C}_{\ell}$ are disjoint if $j \neq \ell$. Therefore

$$
n=\left|\mathcal{C}_{1}\right|+\cdots+\left|\mathcal{C}_{r}\right|+r+1 .
$$

It follows from (28) that $n$ has the same parity as $s+1$. Since $n$ is odd, $s$ must be even. Thus $i$ is adjacent to an even number of vertices $j$ such that $G \backslash\{j\}$ has no odd component. It follows that the coordinate of $A x$ indexed by $i$ is zero. Since $i$ is arbitrary we conclude that $A x=0$.

We now turn to the uniqueness of the null vector. Let $y$ be a vector over $\mathbb{F}_{2}$ such that $A y=0$. If $y \neq x$, then $z=x+y$ is a nonzero vector such that $A z=0$. Moreover, there exists $i$ such that $x_{i}=1$ and $z_{i}=0$. Then $A(i \mid i)$ has a nonzero null vector and hence it must be singular. However $G \backslash\{i\}$ has at most two components, both of which must be nonsingular by Theorem 9 . This contradiction shows that $y=x$ and hence $x$ is the unique null vector of A.

It was shown by Gutman and Sciriha [8] that over the reals, the line graph of a tree is either singular or has zero as a simple eigenvalue. Thus the nullity of the line graph of a tree over the reals is at most one. An alternative proof of the result and some related results were proved in [10]. An easier proof of the same result and some extensions were recently proved in [2],[6]. It may be remarked that in view of Theorem 10, the nullity of the line graph of a tree is at most one over $\mathbb{F}_{2}$ as well. This observation is a special case of a result obtained by Doob [5, Theorem 2.9] and can also be derived easily from some results in [7]. Also, the first part ( $n$ even) of Theorem 10 is contained in [7, Fact 22].

\section{Flower}

A block graph is called a flower if it has at most one cut vertex. By $F\left(b_{1}, \ldots, b_{k}\right)$ we denote a flower with $k$ blocks of sizes $b_{1}, \ldots, b_{k}$. If $k=1$, then $F$ is a complete graph. Although the results in this section apply to this case, we implicitly assume in the proofs that $k \geq 2$, so that $F$ has exactly one cut-vertex. A flower with blocks of sizes $2,2,3,4$ is shown below.

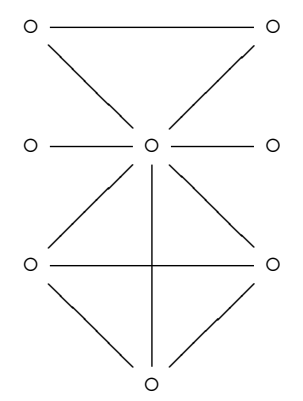


Theorem 11 Let $F$ be a flower with $n$ vertices and with $k$ blocks of sizes $b_{1}, \ldots, b_{k}$. Let $A$ be the adjacency matrix of $F$. Then

$$
\operatorname{det} A=(-1)^{n-k}\left(k \prod_{i=1}^{k}\left(b_{i}-2\right)+\sum_{i=1}^{k} \prod_{j \neq i}\left(b_{j}-2\right)\right) .
$$

Proof: Note that $n=\sum_{i=1}^{n} b_{i}-k+1=\sum_{i=1}^{k}\left(b_{i}-1\right)+1$. Thus if $\left(\alpha_{1}, \ldots, \alpha_{k}\right)$ is a $k$-tuple of nonnegative integers satisfying the conditions $(i),(i i)$ of Theorem 2 , then $\alpha_{i}=b_{i}$ for some $i$ and $\alpha_{j}=b_{j}-1, j \neq i$. The result now follows easily from Theorem 2 .

Theorem 12 A flower is nonsingular over $\mathbb{F}_{2}$ if an only if it has exactly one even block.

Proof: The result may be proved using Theorem 11. It is also an easy consequence of Theorem 6 .

In the final result we determine the nullity of a flower.

Theorem 13 Let $F$ be a flower with $\ell$ even blocks. Let $A$ be the adjacency matrix of $F$. Then the nullity of $A$ over $\mathbb{F}_{2}$ is $|\ell-1|$.

Proof: Let $F$ have $n$ vertices and $k$ blocks of sizes $b_{1}, \ldots, b_{k}$. Then $n=\sum_{i=1}^{k}\left(b_{i}-1\right)+1$. First suppose $\ell=0$. Then $n$ is odd and by Lemma $5, A$ is singular. If we delete a vertex, other than the cut-vertex, from one of the blocks, then the resulting graph is a flower with exactly one even block, and is nonsingular by Theorem 12 . Thus rank $A=n-1$ and hence the nullity of $A$ is 1 . Now let $\ell \geq 1$. Remove one vertex from any $\ell-1$ of the even blocks. The resulting graph is nonsingular by Lemma 5 and hence the rank of $A$ is at least $n-\ell+1$. Since $A$ is symmetric, its rank is $r$ if and only if it has a nonsingular principal submatrix of order $r$. Thus in order to complete the proof we need to show that if we remove any $\ell-2$ vertices from $F$, the resulting graph is singular. Let $H$ be the graph obtained from $G$ by removing some $\ell-2$ vertices. If the cut-vertex is not among the deleted vertices, then $H$ is a flower with at least two even blocks and is singular by Theorem 12. If the cut-vertex is among the deleted vertices, then $H$ must have at least one odd component and again $H$ is singular by Lemma 5 . That completes the proof.

Acknowledgment: We sincerely thank the referee for a careful reading of the paper and for several helpful suggestions. The first author acknowledges support from the JC Bose Fellowship, Department of Science and Technology, Government of India.

\section{References}

1. R. B. Bapat, Graphs and Matrices, Universitext, Springer, London; Hindustan Book Agency, New Delhi, 2010. 
2. R. B. Bapat, A note on singular line graphs, Bulletin of Kerala Mathematics Association, 8(2):207-209 (2011).

3. S. Barik, M. Neumann and S. Pati, On nonsingular trees and a reciprocal eigenvalue property, Linear and Multilinear Algebra, 54(6):453-465 (2006).

4. F. Buckley, L. L. Doty and F. Harary, On graphs with signed inverses, Networks, 18(3):151-157 (1988).

5. M. Doob, An interrelation between line graphs, eigenvalues, and matroids, J. Combinatorial Theory Ser. B, 15:40-50 (1973).

6. E. Ghorbani, Spanning trees and line graph eigenvalues, arXiv:1201.3221v1 (2012).

7. John Goldwasser, Xinmao Wang and Yaokun Wu, Does the lit-only restriction make any difference for the $\sigma$-game and the $\sigma^{+}$-game?, European Journal of Combinatorics, 30:774-787 (2009).

8. I. Gutman and I. Sciriha, On the nullity of line graphs of trees, Discrete Math., 232(13):35-45 (2001).

9. R. A. Horn and C. R. Johnson, Matrix Analysis, Cambridge University Press, 1985.

10. M. C. Marino, I. Sciriha, S. K. Simic, and D. V. Tošić, More about singular line graphs of trees, Publ. Inst. Math. (Beograd) (N.S.) 79(93):1-12 (2006).

11. D. West, Introduction to Graph Theory, Second ed., Prentice-Hall, 2001. 